\title{
Knochenalterbestimmung bei Asylsuchenden ist unbrauchbar
}

\author{
Georg Friedrich Eicha, Valérie Schwitzgebel ${ }^{b}$ \\ a PD Dr. med., Präsident der Schweizerischen Gesellschaft für Pädiatrische Radiologie (SGPR) \\ b Prof. Dr. med., Präsidentin der Schweizerischen Gesellschaft für Pädiatrische Endokrinologie und Diabetologie (SGPED)
}

Die aktuellen politischen Umwälzungen und Kriege auf der Welt, speziell im Nahen Osten und Nordafrika, haben zu bedeutenden Flucht- und Migrationsbewegungen in Europa geführt, von denen auch die Schweiz betroffen ist. Unter den Schutzsuchenden befinden sich viele, teilweise unbegleitete Kinder, Jugendliche und junge Erwachsene, mit oft ungenügender oder fehlender Dokumentation von Identität, Geburtszeit und -ort. Da minderjährige Asylsuchende einen Anspruch auf besonderen Schutz und Beistand haben, ist die Feststellung des Alters (chronologisches Alter) von erheblicher juristischer Bedeutung. Die radiologische Knochenalterbestimmung zur Feststellung der Minder- resp. Volljährigkeit wird deshalb zunehmend von den involvierten Behörden im Asylverfahren angefragt. Eine entsprechende Untersuchung, im Allgemeinen eine Handröntgenaufnahme, wird von Ärzten durchgeführt und meistens mit dem Atlas von Greulich und Pyle [1] beurteilt. Dieses Vorgehen und dessen Ziel sind unserer Meinung nach kritisch zu beurteilen [siehe auch: 2-4]. Wir sind der Meinung, dass radiologische Untersuchungen zur Altersbestimmung bei Asylsuchenden aus folgenden Gründen unterlassen werden sollen:

1. Die Skelettalterbestimmung nach Greulich und Pyle sowie Tanner \& Whitehouse sind anerkannte Standardmethoden zur Bestimmung des biologischen Alters. Sie sind nicht zur Bestimmung des chronologischen Alters vorgesehen. Sie wurden mit dieser Fragestellung auch nicht geprüft.

2. Die individuelle Variabilität (Standardabweichung) des Knochenalters, bestimmt nach Greulich und Pyle, beträgt bei einem 17-jährigen männlichen Jugendlichen beispielsweise 15,4 Monate. Unter Berücksichtigung einer doppelten Standardabweichung, die üblicherweise als Mass für die Normalität in der Medizin gebraucht wird, ergibt sich daraus eine Abweichung von über 2 Jahren. So kann ein gesunder 17-jähriger Knabe durchaus ein Knochenalter von 19 Jahren aufweisen, somit eine abgeschlossene Knochenreifung. Mit anderen Worten: Auch bei vollständigem Schluss der Wachstumsfugen ist ein chronologisches Alter von unter 18 Jahren möglich.
3. Mädchen erreichen den Abschluss des Wachstums früher als Knaben, was die Wahrscheinlichkeit erhöht, dass ein minderjähriges Mädchen ein ausgereiftes Skelett in der Handröntgenaufnahme zeigt.

4. Die Methode von Greulich und Pyle basiert auf einem Normalkollektiv von Kindern europäischer Herkunft aus den USA. Die Studie wurde im frühen 20. Jahrhundert durchgeführt. Aktuelle Daten zum Knochenalter unterschiedlicher Ethnien sind nur punktuell vorhanden und kaum auf die heutige Immigrantenpopulation anwendbar [5].

5. Körperliche Erkrankungen können zu einem Abweichen der Knochenreifung von der Norm führen. Eine Beurteilung der Knochenreifung ohne zusätzliche medizinische Untersuchung der Asylbewerber, speziell im Hinblick auf mögliche Störungen der endokrinen Organe und ohne Beurteilung des Ernährungszustandes ist abzulehnen.

Die Röntgenaufnahme wird mit ionisierenden, potentiell krebsauslösenden Strahlen durchgeführt. Obschon die Strahlendosis für eine Aufnahme der Hand relativ gering ist und einem Bruchteil der natürlich erhaltenen Jahresdosis entspricht, wird diese Untersuchung im klinischen Betrieb aus Sicherheitsüberlegungen nur bei entsprechender medizinischer Indikation durchgeführt. Diese Indikation entfällt bei den Asylsuchenden.

Wir folgern daher, dass die Knochenalterbestimmung bei Asylsuchenden zur Unterscheidung von Minderund Volljährigkeit ethisch bedenklich und methodologisch unbrauchbar ist.

\section{Literatur}

1 Greulich WW, Pyle SI. Radiographic Atlas of Skeletal Development of the Hand and Wrist, 2nd edition. Stanford, CA: Stanford University Press, 1959.

2 Nowotny T, Eisenberg W, Mohnike K. Unbegleitete minderjährige Flüchtlinge - Strittiges Alter - strittige Altersdiagnostik. Deutsches Ärzteblatt 2014; 111(18); A-786-8.

3 Aynsley-Green A, Cole TJ, Crawley H, Lessof N, Boagj LR, Wallace LMM. Medical, statistical, ethical and human rights considerations in the assessment of age in children and young people subject to immigration control. British Medical Bulletin 2012; 1-26.

4 Smith T, Brownlees L. Age assessment practices: a literature review \& annotated bibliography 2011 by United Nations Children's Fund (UNICEF), New York, 2011.

5 Ontell FK, Ivanovic M, Ablin DS, Barlow TW. Bone Age in Children of Diverse Ethnicity. AJR 1996;167; 1395-8. 